\title{
RESEARCH
}

\section{Whole-cell and acellular pertussis vaccination programs and rates of pertussis among infants and young children}

\author{
David Vickers, Allen G. Ross, Raúl C. Mainar-Jaime, Cordell Neudorf, Syed Shah
}

$\infty$

See related article page 1227

\section{ABSTRACT}

Background: The transition from a whole-cell to a 5-component acellular pertussis vaccine provided a unique opportunity to compare the effect that each type of vaccine had on the incidence of pertussis, under routine conditions, among children less than 10 years of age.

Methods: Analyses were based on passive surveillance data collected between 1995 and 2005. The incidence of pertussis by year and birth cohort was compiled according to age during the surveillance period. We determined the association between vaccine type (whole-cell, acellular or a combination of both) and the incidence of pertussis using Poisson regression analysis after controlling for age ( $<1$ year, $1-4$ years and 5-9 years) and vaccination history (i.e., partial or complete).

Results: During 7 of the 11 years surveyed, infants $(<1$ year of age) had the highest incidence of pertussis. Among children born after 1997, when acellular vaccines were introduced, the rates of pertussis were highest among infants and preschool children (1-4 years of age). Poisson regression analysis revealed that, in the group given either the whole-cell vaccine or a combination of both vaccines, the incidence of pertussis was lower among infants and preschool children than among school-aged children ( $5-9$ years). The reverse was true in the group given only an acellular vaccine, with a higher incidence among infants and preschool children than among school-aged children.

Interpretation: These results suggest that current immunization practices may not be adequate in protecting infants and children less than 5 years of age against pertussis. Altering available acellular formulations or adopting immunization practices used in some European countries may increase the clinical effectiveness of routine pertussis vaccination programs among infants and preschool children.

CMAJ 2006;175(I0):1213-7

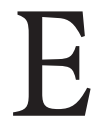
ffective pertussis vaccination programs have reduced the incidence of serious disease globally. However, pertussis continues to pose a threat to infants and young children. ${ }^{1,2}$ The potential for severe side effects after vaccination as well as reported shifts in the age distribution of pertussis to adolescents and adults in North America have led to the replacement of whole-cell pertussis vaccines by a variety of acellular formulations. ${ }^{2}$

Although acellular pertussis vaccines have a higher reported efficacy than whole-cell vaccines, ${ }^{3,4}$ the effect of either type of vaccine on the incidence of pertussis has not been consistent under routine conditions and depends on the type and manufacturer of the vaccine..$^{5-8}$ Analyses of surveillance data in Senegal have shown higher rates of vaccine failure, and subsequently a higher incidence of pertussis, among children given a 2-component acellular vaccine than among those given a whole-cell vaccine (for 3 doses). ${ }^{6,7}$ Research in Germany has also shown higher incidence rates among children given a 4component acellular vaccine than among those given a wholecell vaccine (0.27 v. 0.19 cases per Ioo person-years). ${ }^{9}$

To our knowledge, the full impact of acellular vaccines on the incidence of pertussis at the population level has been studied intermittently and remains to be established. ${ }^{10-12}$ Investigating the incidence of pertussis before and after the introduction of an acellular vaccine presents an excellent opportunity to compare the effectiveness of the 2 types of vaccine in controlling pertussis. We analyzed passive surveillance data before and after such a transition to compare the effect of whole-cell vaccine and a 5-component acellular vaccine on the incidence of pertussis among infants and children aged less than io years, after controlling for other pertussisrelated risk factors.

\section{Methods}

Pertussis is a notifiable disease in North America. We used passive surveillance data collected by the Saskatoon Regional Health Authority (SRHA) in Saskatchewan between Jan. I, I995, and Sept. 30, 2005, from an aggregate population of about II2 278 children. Children who met the clinical criteria for pertussis (i.e., $>2$ weeks with paroxysmal cough, cough with gagging, vomiting, apnea or inspiratory "whoop") or who were known to have had contact with a clinical case had nasopharyngeal specimens taken by swab when they visited the doctor. Pertussis infection was confirmed by means of polymerase chain reaction (PCR) assay of the nasopharyngeal specimens.

We included all pertussis cases in the SRHA database reported during the surveillance period and analyzed them by age-specific incidence and birth cohort. Stratified investiga- 
tions by vaccine type and vaccination history required that subsequent analyses be continued in a multivariable context. Therefore, we used a Poisson regression model to measure the association between vaccine type and the incidence of pertussis after controlling for age and vaccination history. The ages of the cases were collapsed into 3 categories (< I year, I -4 years and 5-9 years); the exact ages of infants less than I year old were not available from these data. Vaccination history was defined as partial (vaccination records indicated partial or lapsed vaccination for the child's age) or complete

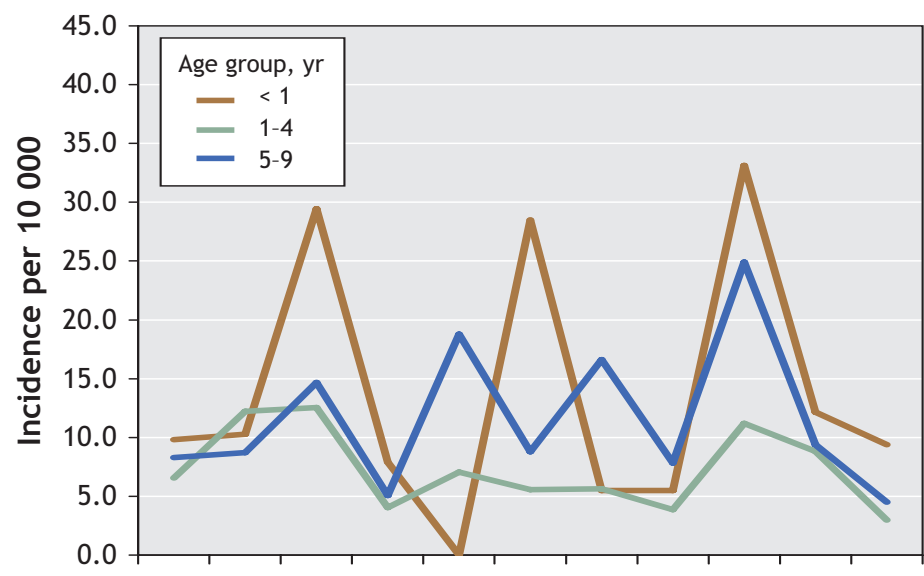

19951996199719981999200020012002200320042005

Fig. 1: Age-specific incidence of pertussis (per 10000 population) among children less than 10 years old in Saskatoon Regional Health Authority (1995-2005), by age group.

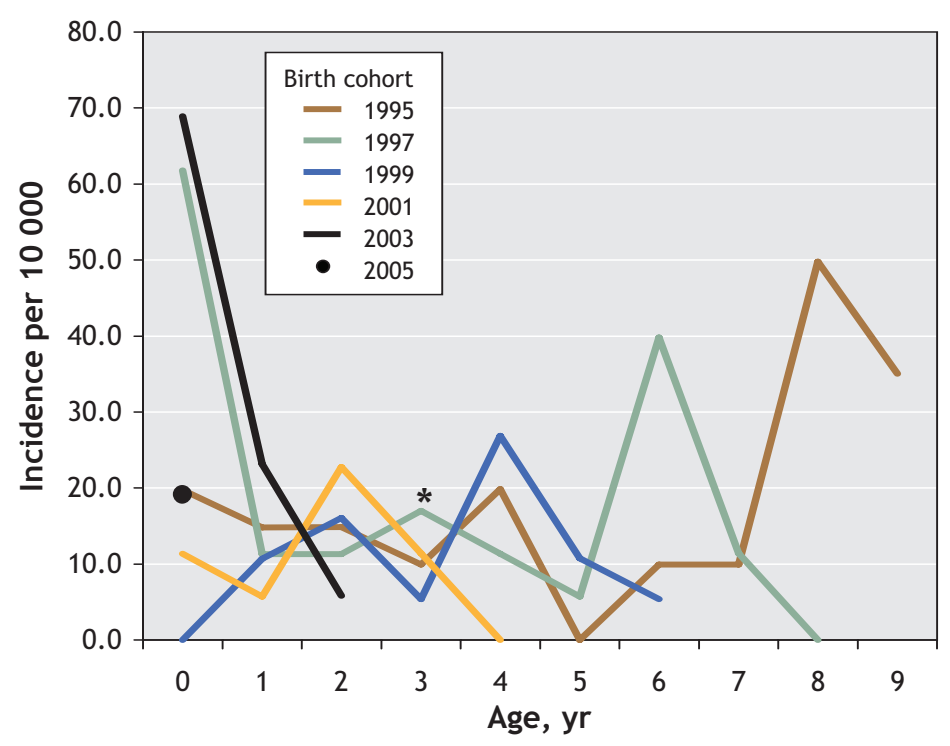

Fig. 2: Age-specific incidence of pertussis (per 10000 population) among children less than 10 years old in Saskatoon Regional Health Authority, by birth cohort (each year represents the year in which members of the cohort were < 1 year old). ${ }^{*}$ The absolute incidence of pertussis at age 3 years for the cohort born in 2000 (data not shown) was estimated to be 32.2 per 10000 population. (child received at least 4 doses or age-appropriate doses of pertussis antigen). Vaccine type was defined as whole-cell (Penta, Sanofi Pasteur Canada), acellular (Quadracel or Pentacel, Sanofi Pasteur Canada) or a combination of both (initial vaccination with whole-cell vaccine followed by booster doses with an acellular formulation). ${ }^{13}$

We were unable to obtain reliable electronic vaccination records for the entire study period. Therefore, denominators for the incidence of pertussis were based on unique combinations of age group, vaccine type and vaccination history from annual population data for children aged less than I year, I-4 years and 5-9 years in the SRHA. Because the type of vaccine changed over the surveillance period, we considered children at risk of pertussis receiving each type of vaccine, already categorized by age, to be the average population of children in a period during which birth cohorts would have theoretically received a given type of vaccine (method adapted from Szklo and Nieto $^{14}$ ). Therefore, we estimated the number of children at risk by calculating the average population aged less than I year and I-4 years during 1990-I99I and 1990-I994, respectively, for the whole-cell vaccine; during 1992-I997 and 1995-1999, respectively, for the combination of whole-cell and acellular vaccines; and during I9982005 and 200I-2005, respectively, for the acellular vaccine. For children aged 5-9 years, the average population of children at risk were those in that age group during 1990-1996 for the whole-cell vaccine, during 1997-2002 for the combination of vaccines and during 2003-2005 for the acellular vaccine.

Childhood immunization against pertussis routinely consists of 3 doses given at 2, 4 and 6 months of age, a fourth dose at 18 months and a fifth dose between 4 and 6 years of age. ${ }^{1}$ The average vaccination coverage rate among children aged 7 years in the SRHA, between 200I-2003, was assumed to represent the proportion of children with a complete vaccination history for the cases in the data set (about 84.I\%). The remaining proportion was assumed to represent those with partial coverage (about $15.9 \%$ ). Information on children with no vaccination history was not available in the data set. We applied these vaccination coverage rates to the average population of children for each combination of age group and vaccine type to obtain the denominators used to estimate the incidence of pertussis.

For the Poisson regression analysis, we assessed the effect of vaccine type on the incidence of pertussis (after adjusting for age group and vaccination history) through partial deviances, or scaled partial deviances (spDEV) in the presence of overdispersion (at $\alpha=$ o.Io). ${ }^{15}$ Because age and vaccination history have previously been associated with the incidence of pertussis, ${ }^{1}$ they were retained in the model as potential confounders regardless of their significance. ${ }^{15}$ Pearson's $\chi^{2}$, or scaled $\chi^{2}$, determined model fit. The exponentiation of the Poisson regression coefficients provided 
an estimate of the adjusted rate ratios (RRs) comparing cases in different risk categories.

\section{Results}

PCR assay identified 472 cases of pertussis. We included 360 of these cases in the multivariable analysis because they had information on all of the variables (age, vaccine type and vaccination history). Children aged 5-9 years, those who received the whole-cell pertussis vaccine and those with a complete vaccination history accounted for the majority of cases over the study period $(67.8 \%, 60.3 \%$ and $88.9 \%$ respectively).

The absolute incidence of pertussis among children aged less than ro years was highest among infants (< I year) (Fig. I). Except for 1999 and 200I, children less than I year and I-4 years experienced the highest rates of pertussis. The introduction of the acellular vaccine in 1997 did not appear to be associated with a reduction in the incidence of pertussis among school-aged children (5-9 years) as expected. ${ }^{16}$

Under an assumption of no severe in-and-out migration, we also plotted the incidence of pertussis by age and birth cohort. ${ }^{17}$ Fig. 2 shows the age-specific incidence by birth cohort in the SRHA for odd-numbered years between 1995 and 2005 . Overall, the graph reveals no distinctive pattern across successive cohorts. The highest incidence through successive childhood ages (i.e., o-4 years) occurred in birth cohorts that would have received only the acellular vaccine. More specifically, the highest age-specific incidence rates occurred among infants less than I year and children $\mathrm{I}-4$ years in the 2003 cohort. In addition, in the 200I, 2000 (not shown) and I999 birth cohorts, the highest age-specific incidence rates were among children aged 2, 3 and 4 years, respectively.
Table $\mathrm{I}$ and Fig. 3 present the results of the Poisson regression analysis. By including an interaction term between age group and vaccine type, we provided additional information to the main effects model $\left(\operatorname{spDEV} \chi^{2}=20.90,4\right.$ degrees of freedom [df], $p<0.0 \mathrm{I}$ ) while maintaining a good fit with the data (scaled Pearson's goodness-of-fit $\chi^{2}=26.00,26 \mathrm{df}, 0.50$ $<p<0.60$ ). Among the children who received only the acellular vaccine, infants and preschool children had higher incidence rates than children aged 5-9 years (RR 8.40, 90\% confidence interval [CI] 3.97-I7.77; and RR 2.48, 90\% CI I.20-5.I2, respectively; Fig. 3). The reverse trend was observed among infants and preschool children given the whole-cell vaccine (RR 0.I7, 90\% CI 0.06-0.47; and RR 0.38, 90\% CI 0.26-0.56; Fig. 3) and among preschool children given both vaccines (RR 0.I6, 90\% CI 0.07-0.36; Fig. 3). Because of small cell sizes among infants in the combined vaccine category, RRs possessed large variance estimates and were hereafter omitted from our discussion.

\section{Interpretation}

Although many studies have shown the efficacy of pertussis vaccines, to our knowledge few have described how the different types of vaccine affect the incidence of pertussis under routine conditions. The results of our descriptive study have associated age and vaccine type with the incidence of pertussis in a population of children less than ro years of age.

Inactivated fractionated pertussis vaccines such as acellular vaccines were introduced into routine vaccination programs because of their improved side-effect profile and comparable immunogenicity when tested against previously used whole-cell pertussis vaccines. ${ }^{5,18-20}$ However, our results sug-

Table 1: Effect of age, vaccine type and vaccination history on incidence of pertussis among children less than 10 years of age in the Saskatoon Regional Health Authority (1995-2005), ${ }^{*}$ by Poisson regression analysis $\dagger$

\begin{tabular}{|c|c|c|c|c|}
\hline Variable & $\begin{array}{c}\text { No. (\%) of cases } \\
n=360\end{array}$ & b coefficient (SE) & RR $(90 \% \mathrm{Cl})$ & $p$ value \\
\hline \multicolumn{5}{|l|}{ Age group, yr } \\
\hline$<1$ & $30 \quad(8.3)$ & $2.13(0.46)$ & 8.39 (3.97-17.77) & $<0.01 \S$ \\
\hline $1-4$ & $86(23.9)$ & $0.91(0.44)$ & $2.48(1.20-5.12)$ & $0.03 \S$ \\
\hline 5-9‡ & $244(67.8)$ & - & - & - \\
\hline \multicolumn{5}{|l|}{ Vaccine type } \\
\hline Whole-cell & $219(60.3)$ & $2.22(0.38)$ & 9.22 (4.98-17.05) & $<0.01 \S$ \\
\hline Whole-cell and acellular & $63(16.9)$ & $1.30(0.40)$ & $3.66(1.91-7.02)$ & $<0.01 \S$ \\
\hline Acellularł & $190(22.8)$ & - & - & - \\
\hline \multicolumn{5}{|l|}{ Vaccination history } \\
\hline Partial & $46(11.1)$ & $-0.42(0.23)$ & $0.66(0.45-0.96)$ & 0.06 \\
\hline Complete $\neq$ & $426(88.9)$ & - & - & - \\
\hline
\end{tabular}

Note: $\mathrm{SE}=$ standard error, $\mathrm{RR}=$ rate ratio, $\mathrm{Cl}=$ confidence interval.

*About 112278 children were followed through passive surveillance; 472 cases of pertussis were identified, of which 360 had information on all of the variables and were included in the multivariable analysis.

†Log-linear model (Pearson's scaled goodness-of-fit $\chi^{2}=26.0,26$ degrees of freedom; $\left.0.50<p<0.60\right): \ln (y)_{i j k}=b_{0}+b_{1}(a g e)_{i}+b_{2}(v a c c i n e$ type $)_{j}+b_{3}(\text { history })_{k}+b_{4}(\text { age } \times \text { vaccine type })_{i j}+\ln (\text { population })_{i j k}$, where $y=$ the number of reported cases, each $b_{n}$, $=$ the estimated regression coefficient, $i$ and $j=1,2,3$ categories of age and vaccine type, respectively, and $k=1,2$ categories of vaccination history. †Reference category.

§Because of the interaction, the effect of these variables on the incidence of pertussis is interpreted on the basis of the results displayed in Fig. 3. 
gest that, in the short term, some acellular vaccines may not adequately provide full protection against pertussis among infants and children less than 5 years old. This may be a consequence of the type of immunity generated by these vaccines (i.e., higher levels of humoural immunity, or Th2-like responses). ${ }^{18,21,22}$ As a result, children require additional doses to reach adequate levels of immunity. ${ }^{18}$ Whole-cell vaccines, on the other hand, tend to elicit higher levels of cellmediated immunity (ThI-like responses). ${ }^{18,21,22}$ These reasons may explain in part why the highest incidence of pertussis among infants and preschool children occurred in the birth cohorts that would have received only the acellular vaccine, and why children aged less than 5 years who received the whole-cell vaccine early in their immunization schedule had lower rates of pertussis (Figs. 2 and 3). These results may significantly alter perceptions about the public health importance of generating cell-mediated immunity during early childhood.

The acellular vaccine did not appear to be reducing the incidence of pertussis among children aged 5-9 years who received booster doses with the acellular vaccine before starting school (Fig. I). Acellular vaccines given as boosters to children aged $15-20$ months $^{19}$ and $4-6$ years $^{20}$ have also been reported to elicit more diverse immunologic responses than either whole-cell or acellular vaccines alone. ${ }^{19,20}$ Our results help to support these findings, since children aged I-4 years who received both vaccines experienced lower incidence rates than children of the same age who received either the wholecell or acellular vaccine alone.

Whole-cell pertussis vaccines are associated with an increased risk of local adverse reactions. However, previous research has shown that children aged ${ }^{5}-20$ months $^{19}$ and $5-6$ years ${ }^{23}$ who received booster doses with an acellular vaccine ${ }^{19}$ after receiving primary immunization with a whole-cell vaccine experienced as many or fewer side effects at the injection

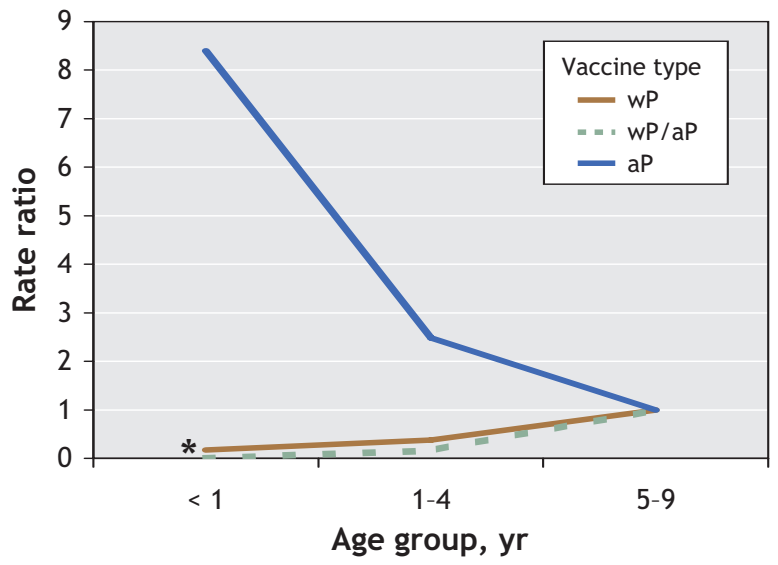

Fig. 3: Effect of vaccine type on the incidence of pertussis among children aged $<1$ year, $1-4$ years and 5-9 years. Note: $w P=$ whole-cell pertussis vaccine, aP = acellular pertussis vaccine, $\mathrm{wP} / \mathrm{aP}=$ combination of both vaccines. ( ${ }^{\star}$ Because of a high variance estimate, the rate ratio for the WP/aP vaccine group has not been interpreted and has only been included to maintain continuity in the figure.) site than did children given an acellular vaccine alone. From the SRHA data, we could not determine when, in their routine immunization, cases in the combined vaccine category began receiving the acellular vaccine. For this reason, further assessment is needed to generate optimal vaccination schedules that not only include both types of vaccine but also determine when to substitute vaccine types in the schedule (to keep adverse events to a minimum).

Evidence of an antigenic shift of virulence factors (i.e., pertussis toxin, pertactin and fimbriae 2 and 3 ) in circulating isolates of Bordetella pertussis has been reported elsewhere. ${ }^{24,25}$ Although selective pressures created by vaccination are thought to be responsible for the observed shift of these bacterial components, no study to our knowledge has specifically identified a correlation between antigenic divergence and an increased incidence of pertussis. If future research provides evidence to support an association between higher incidence rates and antigenic divergence, then the use of both whole-cell and acellular vaccines may better protect children against a greater variety of $B$. pertussis strains that may be circulating in a population.

Our study had a number of limitations. First, we used passive surveillance data. Although such data do not represent the actual number of cases, they can reveal changes in the incidence of pertussis over time, ${ }^{26}$ especially since reporting practices in the SRHA have remained consistent over the surveillance period. Second, reliable electronic data on vaccinerelated adverse events before the year 2000 were not readily accessible. Having these rates for the entire surveillance period would have enabled us to identify clusters of rare vaccinerelated adverse events within the population of the SRHA. More importantly, we might have been able to perform a retrospective investigation to compare these rates in whole-cell and acellular vaccine programs outside of controlled studies. Although acellular pertussis vaccines are known to possess a better side-effect profile than the whole-cell vaccine, severe local reactions can still occur after receiving booster doses with some acellular vaccines. ${ }^{27}$ For this reason, both regional and national surveillance systems should integrate the surveillance of vaccine-related adverse effects with case report data. Doing so may not only place new demands on data collection through passive surveillance, it may also represent a new role for surveillance data in more rigorous epidemiologic research. ${ }^{18}$ Third, for our category-specific denominators we assumed that all children were at risk of pertussis. Since all children were considered vaccinated, although at different levels (partial or complete), different susceptibilities within these groups are expected to be present, since the actual number of doses of pertussis antigen a child received through vaccination will likely vary. However, we do not think this is a major concern, since the rate of vaccination remained similar over the study period (data not shown) and we controlled for vaccination history in the statistical analysis.

The effectiveness of pertussis vaccines is multifactorial. ${ }^{6}$ Our results suggest that further revisions to current immunization programs to include both whole-cell and acellular pertussis vaccines (similar to programs in France, the Netherlands and the United Kingdom ${ }^{28}$ ) may have important public 
health implications for controlling pertussis among children aged less than 5 years. However, further investigation is needed to confirm, or refute, our results. Given that wholecell vaccines are no longer available in North America, it may be more realistic to suggest that current acellular vaccine formulations for children be augmented to include antigenic components derived from circulating strains of $B$. pertussis. Doing so may help increase the long-term effectiveness of these vaccines. Research focused on creating unique vaccine formulations and routes of delivery will ultimately advance our efforts of controlling pertussis. ${ }^{29-32}$

\section{This article has been peer reviewed.}

From the Department of Applied Research (Vickers [at the time of submission]), College of Medicine, University of Saskatchewan; the Saskatoon Regional Health Authority (Ross, Neudorf); the Department of Community Health and Epidemiology (Ross, Shah), College of Medicine, University of Saskatchewan; and the Department of Microbiology (Mainar-Jaime), Western College of Veterinary Medicine, University of Saskatchewan, Saskatoon, Sask.

\section{Competing interests: None declared.}

Contributors: Allen Ross and Cordell Neudorf facilitated the collection and acquisition of the surveillance data used. David Vickers designed the analysis and, together with Raúl Mainar-Jaime and Syed Shah, contributed to the analysis of the data and interpretation of the results. David Vickers drafted and revised the article for intellectual content. All of the authors made substantial revisions to the intellectual content of the manuscript and approved the final version.

Acknowledgements: We thank Dr. Volker Gerdts and Mr. Josh Lawson for their helpful comments on earlier versions of this paper. We also thank the staff at the Saskatoon Regional Health Authority for their kind assistance in making these data available for study.

The Founding Chairs Graduate Fellowship provided funding for this study. The fellowship was awarded from the Institute of Agricultural, Rural, and Environmental Health, College of Medicine, University of Saskatchewan.

\section{REFERENCES}

I. Public Health Agency of Canada. Pertussis. Vaccine-preventable diseases. Available: www.phac-aspc.gc.ca/im/vpd-mev/pertussis_e.html (accessed 2006 Sept 7).

2. Tan T, Halperin S, Cherry JD, et al. Pertussis immunization in the Global Pertussis Initiative North American region: recommended strategies and implementation considerations. Pediatr Infect Dis J 2005;24:S83-6.

3. Bentsi-Enchill AD, Halperin SA, Scott J, et al. Estimates of the effectiveness of a whole-cell pertussis vaccine from an outbreak in an immunized population. Vaccine $1997 ; 15: 301-6$.

4. Gustafsson L, Hallander HO, Reizenstein E, et al. A controlled trial of a two-component acellular, a five-component acellular, and a whole-cell pertussis vaccine. $N$ Engl J Med I996;334:349-55.

5. Mortimer EA, Cherry JD. Pertussis (whooping cough). In: Gershon AA, Hotez PJ, Katz SL, editors. Krugman's infectious diseases of children. Philadelphia: Mosby; 2004. p. 443-59.

6. Lacombe K, Yam A, Simondon K, et al. Risk factors for acellular and whole-cell pertussis vaccine failure in Senegalese children. Vaccine 2004;23:623-8.

7. Simondon F, Preziosi MP, Yam A, et al. A randomized double-blind trial comparing a two-component acellular to a whole-cell pertussis vaccine in Senegal. Vaccine $1997 ; 15: 1606-\mathrm{I} 2$.

8. Bisgard KM, Rhodes P, Connelly BL, et al. Pertussis vaccine effectiveness among children 6 to 59 months of age in the United States, I998-200I. Pediatrics 2005;II6:e285-94.

9. Lugauer S, Heininger U, Cherry JD, et al. Long-term clinical effectiveness of an acellular pertussis component vaccine and a whole cell pertussis component vaccine. Eur J Pediatr 2002;161:I42-6.

Io. Hviid A, Stellfeld M, Andersen $\mathrm{PH}$, et. al. Impact of routine vaccination with a pertussis toxoid vaccine in Denmark. Vaccine 2004;22:3530-4.

II. Olin P, Gustafsson L, Barreto L, et. al. Declining pertussis incidence in Sweden following the introduction of acellular pertussis vaccine. Vaccine 2003;2I:2015-2I

12. McWha L, MacArthur A, Badiani T, et al. Measuring up: results from the National Immunization Coverage Survey, 2002. Can Commun Dis Rep 2004;30:37-50.

13. Vickers D. The epidemiology and transmission of pertussis in the Saskatoon Regional Health Authority: 1995 to 2003 [thesis]. Saskatoon: University of Saskatchewan; 2005.

I4. Szklo M, Nieto FJ. Measuring disease occurrence. In: Epidemiology: beyond the basics. Gaithersburg (MD): Aspen Publishers, Inc.; 2000. p. 55-87.

I5. Kleinbaum DG, Kupper LL, Muller KE, et al. Poisson regression. In: Kugushev A editor. Applied regression analysis and other multivariate methods. 3 rd ed. Pacific Grove: Duxbury Press; 1998. p. 687-709.

I6. De Serres G, Shadmani R, Boulianne N, et al. Effectiveness of a single dose of acellular pertussis vaccine to prevent pertussis in children primed with pertussis whole cell vaccine. Vaccine 2001;19:3004-8.

17. Newman SC. Age-period-cohort analysis. In: Biostatistical methods in epidemiology. New York: Wiley Interscience; 200I. p. 258-62.

I8. Strathdee SA, Loughlin AM. Vaccines: past, present, and future. In: Nelson KE, Williams CM, Graham NMH, editors. Infectious disease epidemiology. Gaithersburg (MD): Aspen Publishers, Inc.; 200I. p. 255-80.

I9. Pichichero ME, Deloria MA, Rennels $\mathrm{MB}$, et al. A safety and immunogenicity comparison of 12 acellular pertussis vaccines and one whole-cell pertussis vaccine given as a fourth dose in I5- to 20-month-old children. Pediatrics I997;100:772-88.

20. Halperin S, Scheifele D, Barreto L, et al. Comparison of a fifth dose of a fivecomponent acellular or a whole-cell pertussis vaccine in children four to six years of age. Pediatr Infect Dis J I999;I8:772-9.

2I. Lavigne MV, Castro M, Mateo N, et al. Whole-cell Bordetella pertussis vaccine component modulates the mouse immune response to an unrelated soluble antigen. Microbes Infect 2002;4:815-20.

22. Mills KH, Ryan M, McGuirk P, et al. The immunology of Bordetella pertussis infection. Biologicals 1999;27:77.

23. Langue J, Matisse N, Pacoret P, et al. Persistence of antibodies at 5-6 years of age for children who had received a primary series vaccination with a pentavalent whole-cell pertussis vaccine and a first booster with a pentavalent acellular pertussis vaccine: immunogenicity and tolerance of a second booster with a tetravalent acellular vaccine at 5-6 years of age. Vaccine 2004;22:I406-I4.

24. Mäkinen J, Mertsola J, Mooi FR, et al. Bordetella pertussis isolates, Finland. Emerg Infect Dis 2005; 11 :183-4.

25. Mooi FR, van Oirschot H, Heuvelman H, et al. Polymorphism in the Bordetella pertussis virulence factors P.69/pertactin and pertussis toxin in The Netherlands: temporal trends and evidence for vaccine-driven evolution. Infect Immun I998;66:670-5.

26. Giesecke J. Modern infectious disease epidemiology. London (UK): Arnold; 2002.

27. Pentacel [product monograph]. Toronto: Sanofi-Pasteur. Available: www .sanofipasteur.ca/sanofi-pasteur-ca/front/templates/index.jsp?codeRubrique $=53$ \&lang $=$ EN (accessed 2006 Sept 7).

28. Pebody RG, Gay NJ, Giammanco A, et al. The seroepidemiology of Bordetella pertussis infection in Western Europe. Epidemiol Infect 2005;I33:I59-7I.

29. Elahi S, Brownlie R, Korzeniowski J, et al. Infection of newborn piglets with Bordetella pertussis: a new model for pertussis. Infect Immun 2005:73:3636-45.

30. Gerdts V, Tsang C, Griebel PJ, et al. DNA vaccination in utero: a new approach to induce protective immunity in the newborn. Vaccine 2004;22:1717-27.

3I. Gerdts V, Snider M, Brownlie R, et al. Oral DNA vaccination in utero induces mucosal immunity and immune memory in the neonate. JImmunol 2002;168:1877-85.

32. Gerdts V, Babiuk LA, van Drunen Littel-van den Hurk, et al. Fetal immunization by a DNA vaccine delivered into the oral cavity. Nat Med 2000;6:929-32.

Correspondence to: David Vickers, Department of Applied

Research, Royal University Hospital, University of Saskatchewan,

Saskatoon SK $S_{7} N$ oW8; fax 306 966-8774;

david.vickers@usask.ca 